\title{
Covid-19 Vaccine Hesitancy in Nursing Home Staff and the Need for Ongoing Education and Vaccine Access
}

\author{
Dheeraj Mahajan ${ }^{1}$, Lori Porter ${ }^{2}$ and Ammu Thampi Susheela ${ }^{3 *}$ \\ ${ }^{1}$ CIMPAR, SC, 101 Madison Street, Suite 300, Oak Park, IL 60302. \\ ${ }^{2}$ National Association of Health Care Assistants (NAHCA), 6079 Co Ln 299, Carl Junction, MO 64834. \\ ${ }^{3}$ Loyola-MacNeal Hospital, 3249 S Oak Park Ave, Berwyn, IL 60402.
}

*Corresponding Author: Ammu Thampi Susheela, Loyola-MacNeal Hospital, 3249 S Oak Park Ave, Berwyn, IL 60402.

Received date: September 06, 2021; Accepted date: September 16, 2021; Published date: January 04, 2022

Citation: Dheeraj Mahajan, Lori Porter and Ammu Thampi Susheela (2022) Covid-19 Vaccine Hesitancy in Nursing Home Staff and the Need for Ongoing Education and Vaccine Access. Clinical Medical Reviews and Reports. 4(1); DOI: 10.31579/2690-8794/103

Copyright: (C) 2022, Ammu Thampi Susheela, This is an open access article distributed under the Creative Commons Attribution License, which permits unrestricted use, distribution, and reproduction in any medium, provided the original work is properly cited.

\begin{abstract}
Objective: To study vaccine hesitancy among health care workers who provide direct care in nursing homes and long term care facilities which cater to the most vulnerable population of the community.

Design: This is a cross sectional cohort study

Setting and participants: The study was conducted on the front line healthcare workers who work in long term care facilities.

Methods: A nationwide survey was conducted on the certified nurse assistants on November $16^{\text {th }} 2020$ which reached 7000 survey recipients.

Results: Out of 7000 survey recipients, 3119 responded (45\% response rate). There was $71.6 \%(2,233)$ negative response about taking the covid19 vaccine due to lack of trust and education of information

Conclusions and implications: Our study concluded that the vaccine hesitancy is high among the health care workers of long-term care facility and that ongoing education and interactive dialogue with certified nurse assistants and ongoing access to vaccine is critical as the willingness improves.

Keywords: covid-19, vaccine hesitancy
\end{abstract}

\section{Introduction}

The COVID-19 pandemic has taken an extensive toll on humanity physically, mentally, and financially. Residents of nursing homes and other long-term care facilities (LCTFs) and settings have been severely and disproportionately affected due to advanced age and the presence of multiple and complex underlying chronic conditions, as well as the setting of congregate living in many cases, and the employment of certified nurse assistants who work in multiple facilities with multiple resident groups.

Data from the federal government and the states on novel coronavirus infections and COVID-19 deaths in nursing homes specifically, combined with comprehensive data compiled in non-governmental databases, have documented the virus' toll in this high-risk segment of the long-term care population. As of February 26, 2021, at least 172,000 coronavirus deaths among the residents and staff of nursing homes and other LTCFs were reported by the New York Times database [1]. And according to a nursing home dashboard maintained by the AARP Public Policy Institute and the Scripps Gerontology Center at Miami University in Ohio, there were 9.2 COVID-19 cases--and 1.95 COVID-19 deaths--per 100 nursing home residents in the four weeks ending on January 17, 2021 [2]. In addition to illness and death, the physical separation from other residents, family, and other loved ones has taken an emotional and physical toll on nursing home residents [3].

The development of vaccines for SARS-CoV-2 offers hope for nursing home residents, and a potential path back toward social interaction and an end to COVID-19 hospitalization and mortality. Health care workers and residents of nursing homes and other LTCFs were designated in December 2020 for the initial COVID-10 vaccine allocation phase (Phase 1a) by the Advisory Committee on Immunization Practices on allocation of COVID-19 vaccination [4]. An allocation framework developed earlier 
by the National Academy of Medicine similarly gave nursing homes and LTCFs top priority, assigning health care workers in these care settings to its Phase 1a category, and residents of nursing homes and LTCFs to Phase $1 \mathrm{~b}[5]$.

The impact of immunization is contingent upon vaccine acceptance and uptake. The herd immunity is referred as indirect protection from infection conferred to the susceptible individuals when a sufficiently large number of immune individuals exists in the population. The herd immunity threshold is the point at which the susceptible individuals in a population falls below the threshold needed for transmission [6]. An estimated vaccine coverage of $55 \%$ to $82 \%$ of the population is required to attain the herd immunity needed to protect the vulnerable adult population [7]. Kwok et al published about the critical level of population immunity (Pcrit) required to halt the spread of infection in a population using the formula Pcrit $=1-1 / \mathrm{Rt}$ (where Rt is the effective reproductive number of an ongoing epidemic). Rt is calculated by using the exponential growth rate for daily number of new COVID19 cases and a recent estimate of the serial interval with a mean of 4.7 days +-2.9 days at a 0.05 significance level using a statistical software. Based on these calculations, the Pcrit for United States based was 69.6 [8]. Herd immunity of the population as a result of vaccination or natural selection depends on variations in the homogenous mixing of the individuals and the individuals developing sterilizing immunity [6]. Aschwanden et al reports the maladies of attaining herd immunity by natural selection as it causes catastrophic loss of human lives while the society may not return to normal as observed in Manaus, Brazil and Italy [9]. Hence, herd immunity through vaccination is considered as the most desirable solution to the COVID19 pandemic. For SARS Co-V-2, clinical manifestations are a poor indicator of transmissibility as asymptomatic carriers can be highly infectious. Once the herd immunity threshold is reached, efficacy of the herd immunity depends on strength and duration of the immunity acquired. If immunity is unevenly distributed, clusters of susceptible hosts can create local outbreak. Case fatality rates and overall infection fatality rates are the relevant measures of SARS Co-V-2 [6].

The factors deciding vaccine hesitancy is subjective and largely depends on the individuals' decision-making process. The individual's decisionmaking regarding vaccination is complex and is based on social, emotional, cultural or spiritual, political, and cognitive factors. Vaccine hesitancy has been defined by the World Health Organization Strategic Advisory Group of Experts on Immunization as "the delay in acceptance or refusal of vaccination despite availability of vaccine services." It is influenced by factors such as complacency (perceiving that taking the vaccine is not important), convenience, and confidence about safety and efficacy. Contextual influences, individual and group influences, and vaccine-specific issues each play a role [7].

Among health care workers, nursing home staff have traditionally had lower vaccination rates for influenza compared with health care personnel working in hospitals and ambulatory care settings [10]. The concerns including potential adverse reactions, perceived personal lack of susceptibility to infection and perceived lack of vaccine effectiveness. They also reported a general lack of trust for influenza virus stating that they are not effective, could potentially lower immunity, and not needed for healthy people [11].The COVID-19 pandemic presents additional potential hurdles given the unprecedented speed of vaccine development.

Understanding COVID-19 vaccine hesitancy among the certified nurse assistants (CNAs) who provide much of the care in nursing homes is critically important to reversing the pandemic's trajectory of illness, death, and isolation in these settings.

To ascertain the degree of and reasons for COVID-19 vaccine hesitancy in this population of nursing home caregivers, the National Association of Health Care Assistants (NAHCA) conducted an informal poll in
November 2020 of thousands of CNAs about whether or not they would take the COVID vaccine once it became available, and their reasons for planning to accept or decline the vaccine.

\section{Study Data and Methods}

\section{Study Hypothesis}

Vaccine hesitancy among health care workers is lesser compared to general population.

\section{Setting and sample}

The study was conducted by National Association of Health Care Assistants (NAHCA) which is the only national professional organization that represents certified nurse assistants throughout USA that was found in 1995. The sample population of our study are the certified nursing assistants in the United States. Currently there are around 26,000 registered members in the organization. The study setting was a private group in a social media platform that consists of 14,919 CAN members. The inclusion criteria was subjects registered with national association of health care assistants as certified nurse assistants. Any subjects who are not a CNA were excluded from the study.

\section{Measures/Procedures}

A confidential online survey was conducted on November 16, 2020 and reached 7,000 CNAs through social media platforms of a private group in Facebook in which NHCHA routinely engages. CNAs were asked to respond to the question "Will you take the COVID-19 vaccine?" They were asked to select "yes," "no," or "undecided." They were also asked to explain their reasons in planning to accept or decline vaccination.

\section{Analysis}

The qualitative data answers were tabulated, and their responses were analyzed for major themes (greater than 10 people entering the same responses) and sub-themes (greater than 5 people entering the same responses). The data was stored in a password protected computer.

Three independent study coordinators confirmed the themes and subthemes mentioned in the survey.

The quantitative data were compared to a study that published about vaccine hesitancy in general public around the same time [12] and paired 2 tailed t-test was used to compare that data to measure for statistical significance ( $\mathrm{p}<0.05)$ using Excel software.

\section{Study Results}

Out of the 26,000 registered members, the survey poll reached 7000 recipients $(27 \%$ ). Of 7,000 survey recipients, 3,119 responded (a 45\% response rate). Of the respondents, $2,233(71.6 \%)$ replied no to future COVID-19 vaccination, and 698 (22.4\%) replied yes. The remaining 188 $(6.0 \%)$ were undecided.

The study results was compared to a rapid national assessment of COVID19 vaccine hesitancy in general population that reported out of 1878 population, 1467 was likely to take the vaccine and 411 was unlikely to take the vaccine [12]. A paired 2 tailed t-test using Excel 2016 showed a $\mathrm{p}$ value of 0.88 which was not statistically significant.

Of the reasons provided for planned rejection of a COVID-19 vaccine, a lack of trust and lack of education and information on the vaccine were major themes. Lack of trust on the newly produced vaccine without sufficient clinical trials to validate appears to be major concern among CNAs. Also the lack of education and information regarding the vaccine and herd immunity appears to be another major concern that contribute towards vaccine hesitancy. 
Hesitancy due to the rapidness of the vaccine launches, a lack of information on potential risks and side effects, and a lack of knowledge of the ingredients in the vaccine were among the sub-themes. The speed of vaccine roll out program has generated concerns among the CNA community about the efficacy of the vaccine. The anticipated risks and adverse effects that have not been previously studied along with the lack of knowledge about the ingredients and the mechanism of action of the vaccine as well as the effect on vulnerable population including elderly, pregnant and breast feeding individuals are factors that also contributed to the vaccine hesitancy.

Comments reflecting hesitancy over rapid vaccine development include the following: "Not happening until it's been researched more. I will take it after I see all the long-term effects it has on others, as any vaccine can be deadly to some. I will not be the test dummy."/ "Once it's been on the market and used for at least a year, with clearly documented side effects, [I will] take it." / "I'm not taking a vaccine that I feel was rushed into production. I'll wait and see how it looks after a few years and make my decision then." / "We don't know what the long-term [effects] are going to be from it. And that's something that can't just be undone. I have zero desire to sign up for that."

Some expressed feelings that vaccination was not necessary for protection: "I allow my body to build its [immunities] and fight its own battles the natural way..." / "I am COVID free this far so I must have some good genetics." Others said they wanted to know more about the vaccine: "I want to know what's in it and research those ingredients" / "I would take the vaccine but also would like the information to support the decision and I would want to know what to expect afterward." Some said they would not take the vaccine unless it is mandatory, and others indicated they would quit the profession entirely if COVID-19 vaccination became mandatory.

Most survey respondents who indicated that they would take the vaccine and offered their reasoning explained that their acceptance was due either to experience in already contracting the virus and/or a desire to protect residents. Among their replies: "My residents have been in lockdown for 9 months! 9 LONG months? Don't they deserve to have a light at the end of their tunnel also?" / "At this point the side effects can't be much worse than the COVID I'm living with currently [and] anything to keep my residents safe and get us back to a normal life." /"Absolutely! My residents, community and family are counting on me!" / "I think I would! Due to the fact of having COVID in the past and working in [a hospital] COVID unit."

\section{Discussion}

Hesitancy among CNAs over COVID-19 immunizations is in part unsurprising. Studies conducted during the initial months of the pandemic suggested sizable levels of hesitancy about a future vaccine among health care workers broadly, albeit with gender- and occupation-related discrepancies. Physicians were more likely to indicate acceptance for a COVID-19 vaccine than nurses/nurse assistants [13, 14]. An anonymous survey of 2,047 French healthcare workers conducted from late March through early July, for instance, found that $76.9 \%$ would accept vaccination. Older age, male gender, occupation as a physician, fear about COVID-19, individual perceived risk, and prior flu vaccination were associated with greater acceptance of a future vaccine [14].

Concerns about a novel coronavirus vaccine persisted into the fall of 2020. A survey of health care workers in Los Angeles completed between September 24 and October 16, 2020 found that $66.5 \%$ of 609 participants intended to delay receiving a future vaccine, with evolving SARS-CoV-2 science and expedited vaccine development among the most impactful reasons. Nurses were 4.15 times more likely to indicate intent to delay than doctors [15]. Another study conducted in October-November in France and French-speaking parts of Belgium and Canada showed 48.6\% of health care workers with high acceptance, $23.0 \%$ with moderate acceptance, and $28.4 \%$ with hesitancy or reluctance. Safety of vaccines developed in an emergency was by far the most important factor associated with hesitancy or reluctance [16].

A study of employees across Yale Medicine and Yale New Haven Health system conducted at the time of FDA approval of the Pfizer-BioNTech vaccine, in which 1 in 6 expressed hesitancy about receiving the vaccine, identified 15 themes of reluctance; wanting more follow-up was the most common reason. Others included pregnancy, concern about safety or side effects, wanting to see others get the vaccine, wanting more clinical research and study, wanting to see study results themselves, having an underlying condition not studied, and having severe allergies or prior reactions to vaccines [17].

Also notable in considering CNA hesitancy is the finding in public polls of U.S. adults that there is a lesser likelihood of COVID-19 vaccine acceptance among respondents who are younger, female, and Black [18, 19]. These demographics characterize a sizable portion of the CNA population.

Our survey of CNAs is not the first study to show hesitancy of COVID19 immunization in this population of health care workers. When the Indiana Department of Health surveyed nursing home and assisted living facility staff in its state in mid-November 2020, only $45 \%$ of 8,243 respondents indicated they would be willing to get an FDA-approved COVID-19 vaccine when it became available. Concerns about side effects were the primary reason (70\%) for hesitancy. Other reasons were health concerns, questions about effectiveness, religious reasons, the need for more research, and a lack of trust [20].

Still, our finding that less than one-quarter of CNAs indicated receptiveness to getting a COVID-19 vaccine in November 2020 is surprising in its magnitude and concerning in that CNAs provide the most direct and hands-on care for nursing home residents. The finding that a lack of trust and a lack of education and information on the vaccine were driving much of the vaccine hesitancy in late 2020 indicates that ongoing education, discussion, and vaccination opportunities are essential.

Effective communication about COVID-19 vaccines requires respectful and interactive discussion that considers concerns, provides up-to-date information about vaccine efficacy and safety, and shares the experience of employees who have received their vaccines [17]. Leaders working within facilities to boost CNA vaccination rates can follow expert advice that has been provided for dealing with COVID-19 misinformation more broadly: listen carefully to their concerns, show respect and empathy, refrain from feeling offended by mistrust and misinformation, and celebrate their willingness to have conversations. Redirecting CNAs to trustworthy information sources and explaining what is known medically are important steps to building trust [21].

Our finding of vaccine willingness being tied at least in part to the protection of nursing home residents suggests that highlighting the benefits of immunization to the community, as well as oneself, may also be beneficial.

There may be legal and ethical justification in the future for mandatory COVID-19 vaccination for staff of nursing homes and other long-term care facilities, given that mandatory influenza vaccination requirements for health care workers are supported by some professional medical associations and states. However, under the scenario of emergency use authorization, mandating vaccination is not likely to be widely supported.

Data from the Centers for Disease Control and Prevention show that nursing home residents were getting vaccinated at significantly higher rates than staff at long-term care facilities, particularly nursing homes, during the first month of the CDC's pharmacy partnership program with CVS and Walgreens $[21,22]$. However, there is reason to be hopeful that 
with ongoing attention, CNA acceptance of COVID-19 immunizations will increase over time. of the nursing home staff who were surveyed in mid-November 2020 by the Indiana Department of Health, $44 \%$ of those who were unwilling to take the vaccine upon availability said they would consider it in the future [20, 23]. The NAHCA has periodically and informally polled its member CNAs and has found a $20 \%$ increase in vaccine willingness in early 2021 compared with November 2020 when its poll was conducted.

Our study has several limitations. The study being an observational study conducted via a confidential online survey can induce selection bias as well as reporting bias to our study. The answers were carefully analyzed and the aim of our study was to report the vaccine hesitancy tendencies in health care population. The vaccine hesitancy rate can also differ in different specialties or other sections of health care providers and hence our study cannot be generalized or extrapolated to express view of all health care providers. Besides our poll was conducted in November of 2020 , before the advent of the vaccines and current data. Hence the tendencies of vaccine hesitancy can vary in the current scenarios. Further studies are required to understand the vaccine hesitancy among health care workers and general population and measures to reduce the hesitancy.

Ensuring ongoing availability of the vaccines within facilities so that staff may be vaccinated conveniently and without any barriers once decisions are made is essential.

\section{Conclusion and implications}

This survey of certified nurse assistants, conducted as COVID-19 vaccine availability was anticipated, revealed that approximately 7 in 10 CNAs did not intend to be vaccinated. Ongoing education that is respectful and interactive is needed to address the reasons for their vaccine hesitancy-mainly a lack of trust, concern about potential risks and side effects, and a lack of understanding or full appreciation for the benefits of vaccination to oneself and the community of vulnerable older adults. Ongoing and convenient access to the vaccines will be essential as education continue and willingness to be vaccinated improves.

\section{References}

1. More than one-third of U. S. coronavirus deaths are linked to nursing homes. New York Times 2021 Feb 26.

2. AARP Nursing Home COVID-19 Dashboard: The AARP. 2021 Feb 11 .

3. Centers for Medicare \& Medicaid Services. Memorandum to state survey agency directors on nursing home visitation [Internet]. Ref.:QSO-20-39-NH.

4. Dooling, K, McClung NM, Chamberland M, Marin M, Wallace M, Bell BP, et al. (2020) The Advisory Committee on Immunization Practices' Interim Recommendation for Allocating Initial Supplies of COVID-19 Vaccine-United States, 2020. MMWR, 69(49):1857-59.

5. National Academies of Sciences, Engineering, and Medicine. (2020) Framework for Equitable Allocation of COVID-19 Vaccine. Washington, DC: The National Academies Press.

6. Randolph, H. and Barreiro, L., (2020) Herd Immunity: Understanding COVID-19. Immunity, 52(5), pp.737-741.

7. Al Awaidy ST, Khamis F. (2020) Preparing the Community for a Vaccine against COVID-19. Oman Med J. 35(6):e193.

8. Kwok, K., Lai, F., Wei, W., Wong, S. and Tang, J., (2020) Herd immunity - estimating the level required to halt the COVID-19 epidemics in affected countries. Journal of Infection, 80(6), pp.e32-e33.
9. Aschwanden, C., (2020) The false promise of herd immunity for COVID-19. Nature, 587(7832), pp.26-28.

10. Lane S, MacDonald NE, Marti M, Dumolard L. (2018) Vaccine hesitancy around the globe: Analysis of three years of WHO/UNICEF Joint Reporting Form data 2015-2017. Vaccine; 36(26):3861-7.

11. Frentzel, E., Jump, R., Archbald-Pannone, L., Nace, D., Schweon, S., Gaur, S., Naqvi, F., Pandya, N. and Mercer, W., (2020) Recommendations for Mandatory Influenza Vaccinations for Health Care Personnel from AMDA's Infection Advisory Subcommittee. Journal of the American Medical Directors Association, 21(1), pp.25-28.e2.

12. Khubchandani, J., Sharma, S., Price, J., Wiblishauser, M., Sharma, M. and Webb, F., (2021) COVID-19 Vaccination Hesitancy in the United States: A Rapid National Assessment. Journal of Community Health, 46(2), pp.270-277.

13. Black CL, Yue X, Ball SW, Fink R, Devlin R, Lu P, et al. (2017) Centers for Disease Control and Prevention. Health Care Personnel and Flu Vaccination, Internet Panel Survey, United States, November 2017.

14. Grech V, Gauci C, Agius S. (2020) Vaccine hesitancy among Maltese healthcare workers toward influenza and novel COVID19 vaccination. Early Hum Dev. 10521

15. Gagneux-Brunon A, Detoc M, Bruel S, Tardy B, Rozaire O, Frappe $\mathrm{P}$, et al. (2021) Intention to get vaccinations against COVID-19 in French healthcare workers during the first pandemic wave: A cross sectional survey. J Hosp Infec. 108:168173.

16. Gadoth A, Halbrook M, Martin-Blais R, Gray A, Tobin NH, Ferbas KG, et al. (2020) Assessment of COVID-19 vaccine acceptance among healthcare workers in Los Angeles.medRxiv. 20234468.

17. Verger $\mathrm{P}$, Scronias D, Dauby N, Adedzi, KA, Gobert C, Bergeat M, et al. (2021) Attitudes of healthcare workers toward COVID-19 vaccination: A survey in France and French-speaking parts of Belgium and Canada. Euro Surveill. 26(3):2002047.

18. Roy B, Kumar V, Venkatesh A. (2020) Health care workers' reluctance to take the Covid-19 vaccine: A consumer-marketing approach to identifying and overcoming hesitancy. NEJM Catal Innov Care Deliv. 10.1056/CAT.20.0676.

19. Kreps S, Prasad S, Brownstein JS, Hswen Y, Garibald BT, Zhang B et al. (2020) Factors associated with US adults' likelihood of accepting COVID-19 vaccination. JAMA Netw Open, 3(10):e2025594.

20. Fisher K, Bloomstone S, Walder J, Crawford S, Fouayzi H, Mazor K. (2020) Attitudes toward a potential SARS-CoV-2 vaccine: A survey of U.S. adults. Ann Intern Med. 15:173(12):964-73.

21. Unroe K, Evans R, Weaver L, Rusyniak D, Blackburn J. (2020) Willingness of long-term care staff to receive a COVID-19 vaccine: A single state survey. Journal of the American Geriatrics Society.

22. COVID-19 conspiracies and beyond: How physicians can deal with patients' misinformation. JAMA Network podcast, 2020 Dec 30.

23. Gharpure R, Guo A, Bishnoi CK, Patel U, Gifford D, Tippins A, et al. (2021) Early COVID-19 first-dose vaccination coverage among residents and staff members of skilled nursing facilities participating in the pharmacy partnership for long-term care program-United States, December 2020-January 2021.MMWR, [cited 2021:70(5);178-182]. 
This work is licensed under Creative Commons Attribution 4.0 License

To Submit Your Article Click Here: Submit Manuscript

DOI: $10.31579 / 2690-8794 / 103$
Ready to submit your research? Choose Auctores and benefit from:

$>$ fast, convenient online submission

$>$ rigorous peer review by experienced research in your field

$>$ rapid publication on acceptance

$>$ authors retain copyrights

$>$ unique DOI for all articles

$>$ immediate, unrestricted online access

At Auctores, research is always in progress.

Learn more auctoresonline.org/journals/clinical-medical-reviews-andreports 